\title{
THE SOLITARY WAVE WITH SURFACE TENSION*
}

\author{
BY T. BROOKE BENJAMIN (University of Oxford)
}

1. Introduction. In a recent note on this subject by Shinbrot [1], an approximate calculation leads him to conclude that the solitary wave "is completely obliterated when surface tension is present, if the depth is small enough." This conclusion is incorrect, the origin of the mistake is easy to pinpoint, and a particularly compelling reason to put the record straight can be appreciated. It is just that the effect of surface tension on solitary waves was explicitly and correctly analysed by Korteweg and de Vries in their celebratedbut perhaps not widely enough read!- paper of 1895 [2].

A parameter having critical influence on solitary-wave properties is the number defined by

$$
\tau=T / \rho g h^{2},
$$

where $T$ is the coefficient of surface tension for the liquid, $\rho$ its density, $g$ the gravity force per unit mass and $h$ the depth of the liquid at rest. Shinbrot states in [1], "I show that there can be no solitary wave when $\tau \geq \frac{1}{3}$." In fact, as was originally shown in [2], solitary waves still exist when $\tau>\frac{1}{3}$, but they become waves of depression rather than waves of elevation as they are according to the familiar model with $\tau=0$. Moreover, their speeds $C$ of propagation relative to a state of rest become subcritical (i.e. $C<(g h)^{1 / 2}$ ), rather than supercritical as predicted with $\tau=0$.

The discussion in [1] sets out a scheme of successive approximation, on more or less standard lines, which can in principle be developed to any order. To the order adopted for the presentation of formal results and conclusions therefrom, however, the outcome is in fact precisely equivalent to that following from the Korteweg-de Vries $(\mathrm{KdV})$ equation when its coefficients are properly evaluated for the water-wave problem. An emphatic claim to the contrary is made in Sec. 1 of [1], but it will be shown presently how the KdV equation leads in simple fashion to the same approximate results and thence to the correct interpretation of solitary-wave properties. While this interpretation is more or less complete in practical respects, the general value of higher-order theories for the solitary wave is not, of course, in dispute here. A rational scheme of continued approximation is needed to underpin any solitary-wave model, even if not taken beyond the stage of recovering the classic findings of Rayleigh, Boussinesq and $\mathrm{KdV}$, and such schemes have notably been the basis of constructive existence theories [3,4]. But for reappraisals of the rudimentary model a premium must be put on simplicity.

The following account accordingly attempts a simple summary of the issues, marginally amplifying a previous discussion [5]. A final commentary refers to the physical significance of the present, corrected conclusions about the parameter régime $\tau>1 / 3$.

\footnotetext{
* Received December 22, 1981.
} 
2. Derivation of model equation. Recall first the well-known theory of infinitesimal waves in a uniform layer of liquid subject to surface tension. The theory establishes that the phase speed $c$ of simple-harmonic progressive waves with wavenumber $k(=2 \pi /$ wavelength $)$ is given by the formula

$$
c=c_{0}\left[\left(1+\tau k^{2} h^{2}\right) \frac{\tanh k h}{k h}\right]^{1 / 2},
$$

in which $c_{0}=(g h)^{1 / 2}[6]$. Note that $c(k)$ is an even positive function, as plainly required by the translation invariance of the physical problem, and note that

$$
c=c_{0}\left\{1+\frac{1}{2}\left(\tau-\frac{1}{3}\right) k^{2} h^{2}+O\left(k^{4}\right)\right\}
$$

as $k^{2} \rightarrow 0$. Thus, $c(0)=c_{0}$ is a local maximum if $\tau<1 / 3$, but is a minimum if $\tau>1 / 3$. In fact, if $\tau \geq 1 / 3, c(k)$ is monotonic increasing for all $k>0$, so that the group velocity $c(k)+k c^{\prime}(k)>c(k)$ for all $|k|>0$.

For water at $20^{\circ} \mathrm{C}$, the critical condition $\tau=1 / 3$ corresponds to $h=4.8 \mathrm{~mm}$. As indicated by (2.2), long waves in water of this critical depth suffer virtually no dispersion, which fact is well known [6]. Practical use of it is made in the design of water tables, which exploit the analogy between two-dimensional problems of gas dynamics and geometrically similar problems involving non-dispersive water waves.

The result for simple-harmonic waves may at once be generalized by the Fourier principle. On the hypothesis of one-way propagation in the direction of the horizontal coordinate $x$ increasing, the evolution equation satisfied by the elevation $\eta(x, t)$ of the free surface above its undisturbed level is evidently

$$
\eta_{t}+L \eta_{x}=0,
$$

where $L$ is the symmetric operator whose symbol is the dispersion function $c(k)$. That is, with $\mathscr{F}$ denoting Fourier transformation, $L$ is defined by

$$
L u=\mathscr{F}^{-1}(c \mathscr{F} u)
$$

for $u \in \mathscr{S}(\mathcal{R})$. Note that $L$ is an unbounded (pseudo-differential) operator when $\tau>0$, because then $c(k) \sim(T|k| / \rho)^{1 / 2}$ as $|k| \rightarrow \infty$; but this property has little relevance to the formalities that follow.

An approximate version of (2.3) for moderately long waves is obtained by keeping only the two leading terms of the Taylor series (2.2). Thus, according to the definition (2.4) of $L$, (2.3) gives

$$
\eta_{t}+c_{0} \eta_{x}+\frac{1}{2} c_{0} h^{2}\left(\frac{1}{3}-\tau\right) \eta_{x x x}=0,
$$

which is the linearized form of the $\mathrm{KdV}$ equation. This or some equivalent step is pivotal to every derivation of the $\mathrm{KdV}$ equation. While rigorous justification is difficult, the nature of the formal approximation can, of course, be exhibited more conspicuously by the usual device of stretched coordinates. One takes $x^{\prime}=\varepsilon(x / h)$ and $t^{\prime}=\varepsilon\left(c_{0} t / h\right)$, with $\varepsilon \downarrow 0$ understood in respect of the order-of-magnitude symbol $O$. One then has to suppose that $\eta=H\left(x^{\prime}, t^{\prime}\right)$, where $H$ has the same magnitude as its partial derivatives of every order. It can thus be reckoned that the third term on the left-hand side of $(2.5)$ is $O\left(\varepsilon^{2}\right)$ relative to the first and second terms, and the implicit error in the equation is relatively $O\left(\varepsilon^{4}\right)$.

On the other hand, the classic shallow-water theory [7] ignores dispersive effects that are relatively $O\left(\varepsilon^{2}\right)$ but allows finite displacements $\eta$. It is well known [7] that according to 
this theory the equation for a simple wave propagating in the $x$-direction is, to a first approximation for nonlinear effects on the local wave speed,

$$
\eta_{t}+c_{0}\left\{\eta_{x}+(3 \eta / 2 h) \eta \eta_{x}\right\}=0 .
$$

Supposing that $\eta=O\left(\varepsilon^{2}\right)$, and taking $h$ and $h / c_{0}$ as the units of length and time, we conclude from (2.5) and (2.6) that an approximation allowing consistently for both dispersive and nonlinear effects is the $\mathrm{KdV}$ equation

$$
\eta_{t}+\eta_{x}+\frac{3}{2} \eta \eta_{x}+\frac{1}{2}\left(\frac{1}{3}-\tau\right) \eta_{x x x}=0 .
$$

The relative error is $O\left(\varepsilon^{4}\right)$, and so presumably is negligible if $\varepsilon$ is small enough.

3. Solitary waves. Let $C$ denote the dimensional speed of a solitary wave, so that in the present scheme of units the speed is $F=C / c_{0}$. (This standard definition of Froude number, making it analogous to Mach number in gas dynamics, is preferable to the one adopted in [1]. $F$ here corresponds to $\sqrt{ } F$ there.) Solutions of (2.7) are accordingly sought in the form $\eta(x-F t)$, with $\eta$ and all its derivatives vanishing at $\pm \infty$. After substitution of this form, (2.7) can be integrated to give

$$
2\left(\frac{1}{3}-\tau\right) \eta^{\prime \prime}=4(F-1) \eta-3 \eta^{2},
$$

whence multiplication by $\eta^{\prime}$ and another integration give

$$
\left(\frac{1}{3}-\tau\right)\left(\eta^{\prime}\right)^{2}=2(F-1) \eta^{2}-\eta^{3} .
$$

(This equation is obtained as (4.1) in [1], and the central mistake there can be located in the sentence that ends the paragraph including (4.1).)

Provided $\tau \neq \frac{1}{3}$, (3.1) and (3.2) have solitary-wave solutions

$$
\eta=A \operatorname{sech}^{2} \beta(x-F t)
$$

where the numbers $A, \beta, F$ are related by

$$
A=2(F-1)=4 \beta^{2}\left(\frac{1}{3}-\tau\right) .
$$

The spectrum of solitary waves for each given $\tau \neq \frac{1}{3}$ is describable in terms of a single parameter $\varepsilon>0$, which has precisely the role connoted earlier by $\varepsilon$, being such that the implicit error in (3.3) is $O\left(\varepsilon^{4}\right)$. From (3.4) it is clear that if

$$
A=\varepsilon^{2} \operatorname{sgn}(1-3 \tau),
$$

then

$$
\beta=\varepsilon / 2 \sqrt{ }\left|\frac{1}{3}-\tau\right|, \quad F=1+\frac{1}{2} \varepsilon^{2} \operatorname{sgn}(1-3 \tau) .
$$

Thus, when $0 \leq \tau<\frac{1}{3}$, solitary waves are waves of elevation with supercritical speeds $(F>1)$ determined by the wave amplitude $\varepsilon^{2}$ independently of $\tau$. But when $\tau>\frac{1}{3}$ they are waves of depression with subcritical speeds $(F<1)$, again determined only by $\varepsilon^{2}$. For a given $\varepsilon^{2}$ in either case, the horizontal scale of the solitary wave (e.g. the distance between the two inflexion points in the graph of $\eta$ ) is proportional to $\sqrt{ }|1-3 \tau|$.

In the exceptional case $\tau=\frac{1}{3}$, there is no solitary wave according to the present approximation. The question whether the complete hydrodynamic problem has any such solution in this case remains open. 
4. Commentary. While the curious theoretical facts about solitary waves in the case $\tau>\frac{1}{3}$ have long been on record [2], it appears that little attention has been paid to them. The likely reason for this neglect is that waves on water shallower than $4.8 \mathrm{~mm}$ are strongly affected by viscosity, so that experimental checks on perfect-fluid predictions are generally difficult. I have tried some experiments with layers of water 3 to $4 \mathrm{~mm}$ deep, convincing myself that stable progressive solitary waves of depression are realizable, but they are damped too rapidly for an unequivocal demonstration. Another difficulty is that, in keeping with (2.7) and more generally with the fact noted about group velocity, spurious disturbances arising from the imperfect generation of a solitary wave propagate ahead of the wave when $\tau>\frac{1}{3}$, rather than being left behind as in the familiar case.

Much more impressive evidence of the anomalous properties of long waves when $\tau>\frac{1}{3}$ can be readily seen in the behavior of a positive bore. A rationale for the observed properties of undular bores when surface tension is insignificant was developed by Benjamin and Lighthill [8], whose approach consisted in parameterizing the periodic cnoidal-wave solutions of (2.7) in appropriate terms of energy and momentum. This approach is also applicable to the present case, leading to markedly different conclusions which accord with what is seen. In particular, the gradually varying wave train at the front of a steady bore is in effect reversed from its more familiar, zero- $\tau$ disposition. The shortest and weakest waves appear ahead, and a depression close in form to the solitary wave appears last, being followed by a waveless flow which is subcritical relative to the bore. The final elevation of the free surface is, of course, approximately the constant solution $\eta_{c}=\left(\frac{4}{3}\right)(F-1)$ of $(3.1)$.

Finally, it should be noted that the stability of a solitary wave (3.3) qua solution of (2.7) is not lost when $1-3 \tau$ is changed from positive to negative values. The results of a stability analysis on record [9] are easily appreciated not to be affected by this change. Moreover, the exact problem for solitary waves with surface tension is known [10] to be representable by a variational principle irrespective of the value of $\tau \geq 0$ (minimum total energy for fixed horizontal impulse, which two quantities are invariants of any wave motion), and this attribute of real solitary waves virtually guarantees their stability.

\section{REFERENCES}

[1] Marvin Shinbrot. The solitary wate with surface tension, Quart. Appl. Math. 39, 287-291 (1981)

[2] D. J. Korteweg and G. de Vries, On the change of form of long waves advancing in a rectangular canal and a new type of long stationary waves, Phil. Mag. (5) 39, 422-443 (1895)

[3] K. O. Friedrichs and D. H. Hyers, The existence of solitary waves, Comm. Pure Appl. Math. 7, 517-550 (1954)

[4] A. M. Ter-Krikorov, Théorie exacte des ondes longuess stationnaires dans un liquide hétérogène, J. Mécanique 2, 351-376(1963)

[5] T. Brooke Benjamin, Internal waves of permanent form in fluids of great depth, J. Fluid Mech. 29, 559-592 (1967)

[6] M. J. Lighthill, Waves in fluids, Cambridge University Press, 1978, p. 226

[7] Sir H. Lamb, Hydrodynamics, 6th ed., Cambridge University Press, 1932, $\$ \$ 169,187$

[8] T. Brooke Benjamin and M. J. Lighthill, On cnoidal waves and bores, Proc. Roy. Soc. Lond. A 224, 448-460 (1954)

[9] T. Brooke Benjamin, The stability of solitary waves, Proc. Roy. Soc. Lond. A 328, 153-183 (1972)

[10] T. Brooke Benjamin, Lectures on nonlinear wave motion, Amer. Math. Soc., Lectures in Appl. Math. 15, 3-47 (1974), $\$ 2.2$ 